\title{
APORTACIÓN DE LOS MODELOS ECOHIDROLÓGICOS CON FEEDBACKS AL CONOCIMIENTO DEL FUNCIONAMIENTO DE LOS ECOSISTEMAS DE ZONAS ÁRIDAS Y SEMI-ÁRIDAS
}

\author{
Y. PUEYO* \\ Instituto Pirenaico de Ecología (CSIC), Av. Montañana 1005, P.O. Box 13.034, \\ 50059 Zaragoza, España.
}

\begin{abstract}
RESUMEN. En las zonas áridas y semi-áridas, la redistribución espacial del agua es un proceso fundamental para entender el funcionamiento y la dinámica de los ecosistemas. En estos ecosistemas se da una retroalimentación positiva (feedback) entre la presencia de vegetación y el agua disponible para las plantas, que es responsable de la formación de los patrones espaciales regulares encontrados frecuentemente en estas zonas, y cuyas consecuencias para los ecosistemas han sido extensivamente estudiadas desde la ecohidrología, tanto experimentalmente como de forma teórica. En este artículo revisamos las principales aportaciones que la modelización ecohidrológica que incorpora feedbacks ha proporcionado al conocimiento del funcionamiento de los ecosistemas áridos y semi-áridos, así como las necesidades futuras de estudio en este campo.
\end{abstract}

Contributions of the eco-hydrological models incorporating feedbacks to the knowledge of arid and semi-arid ecosystems functioning

ABSTRACT. Water redistribution is a fundamental mechanism driving the functioning and dynamics of arid and semi-arid ecosystems. There, a positive feedback exists between vegetation biomass and soil water availability for plants, and it is often responsible of a regular spatial pattern development. The consequences of such spatial patterns in plant and water occurrence have been extensively studied in the field of eco-hydrology, both experimentally and theoretically. Here, we review the main contributions of the ecohydrological models incorporating feedbacks to the knowledge of arid and semi-arid ecosystems functioning, as well as the future research needs and directions in this research field.

Palabras clave: retroalimentación positiva, patrón espacial, ecohidrología, modelización ecológica, vegetación, zonas áridas y semi-áridas.

Key words: positive feedback, spatial pattern, eco-hydrology, ecological modelling, vegetation, drylands. 
* Correspondencia: Instituto Pirenaico de Ecología (CSIC), Av. Montañana 1005, P.O. Box 13.034, 50059 Zaragoza, España. E-mail: ypueyo@ipe.csic.es

\section{Introducción: Ecohidrología de los ecosistemas de zonas áridas y semi-áridas}

Las zonas áridas, semi-áridas y subhúmedas ocupan el $41 \%$ de la superficie terrestre (Reynolds et al., 2007), porcentaje que asciende hasta el 74\% en la Península Ibérica (MARM, 2008). En estas zonas habita un tercio de la población mundial, subsistiendo con una economía basada en gran medida en la agricultura y el pastoreo tradicional (Millennium Ecosystem Assessment, 2005). Por ello, el mantenimiento de la productividad y de la diversidad de los recursos pastables es un tema fundamental para la subsistencia y sostenibilidad de estas poblaciones. Para ello, es clave un buen conocimiento del funcionamiento de los ecosistemas pastorales áridos y semi-áridos, que permita una correcta gestión de los recursos y capacidad de predicción frente a cambios futuros de una manera eficaz.

La disponibilidad de agua es el principal recurso limitante que condiciona el funcionamiento de los ecosistemas de las zonas áridas y semi-áridas (Noy-Meir, 1973). En estos ecosistemas, la cantidad de agua disponible para las plantas no sólo depende de la cantidad de precipitación, sino también en gran medida de la redistribución del agua de lluvia dentro del ecosistema (Greigsmith, 1979; Bergkamp et al., 1999; Ludwig et al., 2005). En concreto, la escorrentía superficial, la capacidad de infiltración del suelo, la evaporación y la capacidad de retención del agua por parte del suelo son procesos hidrológicos que van a determinar la cantidad de agua disponible para el establecimiento, viabilidad y productividad vegetal. La capacidad de infiltración en el suelo sin vegetación de las zonas áridas y semi-áridas es a menudo muy escasa, ya que la presencia de costra superficial y la elevada compactación del suelo impiden al agua infiltrarse (Noy-Meir, 1973; Tongway et al., 2001; Hamerlynck et al., 2002). La presencia de costra es un fenómeno frecuente en la superficie de los suelos de las zonas áridas y semi-áridas. Podemos hablar de dos tipos de costra, la costra física, que se origina por el impacto de las gotas de lluvia en la superficie del suelo desnudo, y la costra biológica que la constituyen los líquenes y algas que a menudo colonizan el suelo desnudo de ambientes áridos. La costra física obstruye los poros del suelo, y con ello es responsable de una escasa infiltración y de la generación de escorrentía superficial (Mills et al., 2009). Sin embargo, el papel de la costra biológica en la infiltración no está claro y se han encontrado resultados contradictorios en diferentes trabajos. Hay estudios que ven un incremento de la infiltración en presencia de costra biológica, mientras que otros trabajos constatan una disminución de la infiltración de agua en presencia de costra (West, 1990; Eldridge y Greene, 1994; Eldridge et al., 2000, 2010; Maestre et al., 2002). Parece que el carácter hidrófobo o hidrófilo de las especies integrantes de la costra bióloga van a determinar el papel que juegan en la infiltración de agua en el suelo (Eldridge et al., 2002). Por su parte, una elevada compactación del suelo es una característica intrínseca de algunos tipos de suelo, como las arcillas (Hamerlynck et al., 2002; Moret et al., 2011). También hay factores extrínsecos al ecosistema que pueden agudizar la falta de infiltración. En concreto, el pastoreo, o 
más bien, el sobrepastoreo, es decir, un uso excesivo de los recursos pastables, afecta al ecosistema de varias formas, entre las que se incluye el efecto de pisoteo del suelo, que produce una compactación que tiene como consecuencia la disminución de la capacidad de infiltración del suelo (Castellano y Valone, 2007; Dunne et al., 2011). Esto genera escorrentía superficial, que además de suponer una pérdida de agua ladera abajo, suele llevar asociados procesos de erosión.

La capacidad de infiltración del suelo se ve incrementada en gran medida por la presencia de vegetación (Bhark y Small, 2003; Ludwig et al., 2005; Arnau-Rosalen et al., 2008). Las plantas crean macroporos en el suelo, que permiten que el agua se infiltre, y la textura del suelo se ve muy mejorada por la elevada concentración de materia orgánica que hay debajo de las plantas, que lleva a una mayor capacidad de retención (Joffre y Rambal, 1993; Bochet et al., 1999; Maestre et al., 2002, 2003; Bedfor y Small, 2008; Segoli et al., 2008). No sólo la infiltración es mayor bajo las plantas, sino que la evaporación, una de las mayores pérdidas de agua en los ecosistemas áridos y semi-áridos (Huxman et al., 2005), se ve reducida en gran medida (Callaway, 2007).

Las zonas áridas y semi-áridas se caracterizan por una cubierta vegetal que no es continua, sino distribuida en manchas de vegetación que aparecen alternadas con zonas de suelo desnudo (Fig. 1). Con esta configuración espacial, los procesos de infiltración y escorrentía superficial están fuertemente ligados a la distribución espacial de la vegetación (Tongway et al., 2001). En las zonas desnudas el agua de la lluvia no se infiltra y se genera una escorrentía superficial que, cuando encuentra una zona con vegetación, se infiltra en el suelo. A estos ecosistemas se les conoce como ecosistemas source-sink en la literatura (Cerdà, 1997), y su dinámica está ampliamente estudiada (Cerdà, 1997; Puigdefábregas et al., 1999; Ludwig et al., 2005; Bautista et al., 2007). Por tanto, en estos sistemas, la presencia de vegetación favorece una mayor disponibilidad de agua localmente. A su vez, la disponibilidad de agua favorece el establecimiento de nuevas plántulas y la supervivencia y crecimiento de la vegetación. Es lo que se conoce como un proceso de retroalimentación positiva (feedback) entre la presencia de vegetación y la disponibilidad de agua para las plantas (Rietkerk et al., 2004). Las implicaciones de la presencia de feedbacks en el funcionamiento de los ecosistemas están ampliamente documentadas. En primer lugar, este tipo de feedback entre la presencia de vegetación y la infiltración de agua en el suelo es un mecanismo estabilizador en los ecosistemas de zonas áridas y semi-áridas, que controla la transición de un estado a otro, es decir, el cambio en estructura y función del ecosistema (Turnbull et al., 2012). La concentración de recursos que supone el feedback, hace que el sistema sea capaz de mantener un estado con vegetación en unas condiciones de aridez que una distribución homogénea de agua no permitiría (Rietkerk et al., 2004). Además, el sistema es capaz de sostener una mayor biomasa vegetal y diversidad de especies (Sachak et al., 1998; Rietkerk et al., 2002). Sin embargo, la presencia de feedbacks también confiere estabilidad al estado degradado del ecosistema (Suding et al., 2004; Pueyo et al., 2009). Una vez se ha traspasado un umbral de degradación, la eliminación de la cubierta vegetal conduce a un estado degradado en el que la ausencia de vegetación impide la infiltración de agua, 

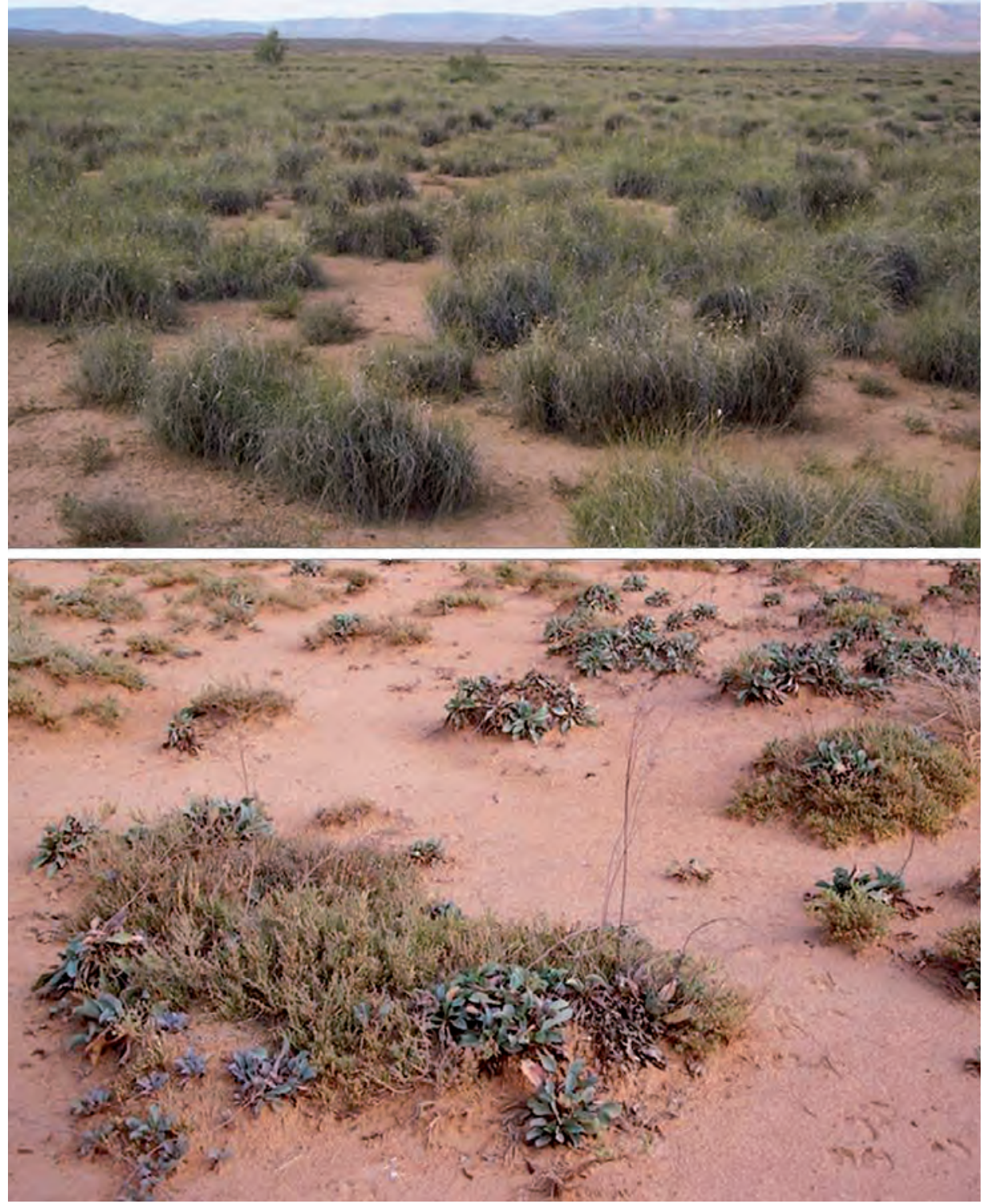

Figura 1. Fotografías de comunidades vegetales en la Reserva Ornitológica del Planerón en la que se aprecia el patrón espacial que forma la alternancia de manchas de vegetación y suelo desnudo. 
lo que a su vez, impide el establecimiento de la vegetación. Asociados a este feedback, aparecen otros, como la degradación del suelo por erosión, que también contribuyen a la estabilidad del estado degradado (Turnbull et al., 2012). Es en estas condiciones cuando el sistema no va a ser capaz de recuperarse por sí sólo, y será necesario el conocimiento del funcionamiento eco-hidrológico del ecosistema para promover una restauración ecológica que lleve al sistema al estado óptimo de conservación (Sunding et al., 2004; Pueyo et al., 2009).

Por tanto, hemos visto que la dinámica source-sink de escorrentía-infiltración de agua que predomina en las zonas áridas y semi-áridas se debe a la distribución de la vegetación en manchas entre zonas de suelo desnudo. A su vez, el patrón espacial de la vegetación se mantiene por la retroalimentación entre presencia de agua y establecimiento vegetal que se establece en las zonas con vegetación. Por ello, el patrón espacial de la vegetación, que es al mismo tiempo origen y propiedad emergente de la dinámica ecohidrológica del sistema, se considera un buen indicador de los procesos subyacentes en el ecosistema, de su estado de conservación y de la posibilidad de un colapso en el ecosistema que lleve a un estado degradado estable (Kéfi et al., 2007a; Zeng y Zeng, 2007). En este último punto, ha habido una controversia recientemente sobre la idoneidad del patrón espacial como indicador temprano del colapso del ecosistema (Maestre y Escudero, 2009; Kéfi et al., 2010) y todavía hay mucho que estudiar para unir los resultados más teóricos con los estudios de campo.

Recientemente está teniendo un gran desarrollo la ecohidrología como una nueva disciplina en la frontera entre la ecología y la hidrología. La ecohidrología es la ciencia que estudia las interacciones recíprocas entre el ciclo hidrológico y los ecosistemas (Rodríguez-Iturbe y Porporato, 2004), y que intenta entender cómo los mecanismos hidrológicos explican los procesos y los patrones ecológicos (Rodríguez-Iturbe et al., 2001). Dentro del campo de la ecohidrología la modelización es una herramienta muy útil en el estudio de los ecosistemas de zonas áridas dominados por feedbacks, ya que éstos son sistemas complejos que se benefician de la capacidad de los modelos de tener en cuenta varios procesos simultáneamente, y de su dinamismo.

El objetivo de este trabajo es revisar los avances en la ecohidrología de las zonas áridas y semi-áridas dominadas por feedbacks, poniendo especial énfasis en los modelos ecohidrológicos que se han desarrollado, su relación con los estudios de campo, las preguntas que contestan y las que plantean para el conocimiento del funcionamiento de los sistemas ecohidrológicos limitados por la disponibilidad de agua. Por tanto, incluyo en esta revisión en los modelos ecohidrológicos que se centran, o al menos incluyen, el feedback entre la presencia de vegetación y la infiltración de agua. Se excluyen de esta revisión los numerosos modelos ecohidrológicos que, aunque incluyen la vegetación como un factor a tener en cuenta en el balance hídrico (por ej., Paruelo y Sala, 1995; Rodríguez-Iturbe et al., 2001), no incluyen el mecanismo de feedback entre la infiltración y la presencia de vegetación.

Esta revisión no se centra en los detalles matemáticos de los diferentes modelos, sino en las principales aportaciones de cada uno de ellos al conocimiento del funciona- 
miento ecohidrológico de las zonas áridas y semi-áridas. Para la comparación de las formulaciones matemáticas de los diferentes modelos se remite a lo largo del texto a los artículos originales, donde los autores detallan sus respectivos modelos (ver también Borgogno et al. 2009 para una completa revisión de las características matemáticas de los modelos orientados a la formación de patrones).

\section{Modelos ecohidrológicos incorporando feedbacks de zonas áridas y semi-áridas}

Muchos de los modelos ecohidrológicos basados en feedbacks de zonas áridas y semi-áridas se centran en explicar los mecanismos de formación de los patrones espaciales auto-organizados de la vegetación (Borgogno et al., 2009). El mecanismo modelizado como responsable de la formación de patrones espaciales es la presencia de un feedback dependiente de la escala: existe un feedback positivo en un rango corto, y un feedback negativo en un rango más largo (Lejeune et al., 1999; Rietkerk et al., 2004; Rietkerk y Van de Koppel, 2008). El feedback dependiente de la escala es un mecanismo de generación de patrones muy extendido en la naturaleza conocido como inestabilidad de Turing, ya que fue Alan Turing quien describió por primera vez cómo dos reactivos con diferentes difusividades podían generar patrones (Turing, 1990). Este mecanismo permite explicar la formación de multitud de patrones en procesos químicos, físicos y de morfogénesis biológica, como por ejemplo, la formación de las manchas en la piel de mamíferos (Turing, 1990; Murray, 2001). Traducido a términos ecohidrológicos, este mecanismo implica que las plantas favorecen la presencia de una mayor disponibilidad de agua localmente, lo que permite instalarse a nuevas plantas en su entorno (feedback positivo), pero a mayor escala, las plantas disminuyen la disponibilidad de agua en la comunidad vegetal, por la competencia que ejercen por el agua como recurso limitante (feedback negativo). Además, frecuentemente la zona radicular de las plantas se extiende más allá de la parte aérea, lo que supone que el efecto positivo de la presencia de plantas sobre la disponibilidad de agua (infiltración y menor evaporación) se da a una escala menor que el efecto negativo por consumo de agua (Couteron y Lejeune, 2001; Gilad et al., 2004; Barbier et al., 2008). Los modelos deterministas que incluyen este mecanismo son capaces de reproducir los patrones espaciales regulares encontrados en la naturaleza (bandas, laberintos y manchas circulares; Fig. 1).

Uno de los primeros modelos sobre formación de patrones regulares como consecuencia de un feedback dependiente de la escala entre la presencia de vegetación y la disponibilidad de agua fue el de Thiery et al. (1995). Aunque este modelo es fenomenológico, es decir, no incluía el movimiento del agua explícitamente, fue un gran avance para comprender el mecanismo subyacente en los patrones espaciales en forma de bandas perpendiculares a la pendiente encontrados en laderas de zonas semi-áridas (Valentin et al., 1999). Mediante un modelo estocástico de autómatas celulares, se reprodujo la formación y dinámica ladera arriba de las bandas de vegetación con el único mecanismo de una instalación preferencial de la vegetación en el borde ladera arriba de las bandas, donde se infiltra el agua de escorrentía superficial generada en el espacio entre bandas inmediatamente anterior, y una mortalidad preferencial de la 
vegetación en el borde ladera abajo de las bandas, debido a la escasez de agua por el consumo del agua de escorrentía por parte de la banda de vegetación superior (Fig. 2a). Posteriormente, se investigó la formación y dinámica de estos patrones espaciales de biomasa vegetal mediante ecuaciones diferenciales (que permitía variables continuas en el espacio y en el tiempo) mediante el feedback dependiente de la escala, denominado en estos trabajos "cooperación a escala corta e inhibición a escala larga” (Lefever y Lejeune, 1997; Lejeune et al., 1999; Lejeune y Tlidi, 1999). Simultáneamente, Klausmeir (1999) introdujo explícitamente la dinámica del agua en el modelo, corroborando el mismo mecanismo de formación de patrones en banda en laderas. Este modelo consiste en un sistema de dos ecuaciones diferenciales parciales, agua disponible en el suelo y biomasa vegetal. El agua se mueve ladera abajo por un proceso de convección, hasta que encuentra la banda de vegetación, donde se infiltra. A su vez, la biomasa vegetal aumenta directamente con la presencia de agua, que es máxima en la parte de la banda de la vegetación localizada ladera arriba, ya que recibe el agua de la escorrentía de la zona sin vegetación, y mínima en la parte de la banda de vegetación que queda ladera abajo, donde se produce la disminución de la biomasa vegetal por ausencia de agua (Fig. 2a). Diversos modelos han comprobado que el mismo mecanismo (Fig. 2b) opera en zonas sin pendiente (Couteron y Lejeune, 2001; Von Hardenberg et al., 2001; Rietkerk et al., 2004), reproduciendo los patrones en puntos y laberintos ubicuos en la naturaleza en estas mismas condiciones (Rietkerk y Van de Koppel, 2008). Estas primeras aproximaciones matemáticas que se hicieron más o menos simultáneamente para explicar la formación de patrones en zonas áridas obtuvieron unos resultados muy similares, lo que da robustez a las asunciones de estos modelos, y a las conclusiones y predicciones obtenidas con ellos.

Un avance significativo hacia modelos más mecanicistas que reproducen los patrones espaciales de la vegetación mediante el feedback agua-biomasa vegetal fue incluir explícitamente la escorrentía superficial en la formulación matemática (HilleRisLambers et al., 2001; Rietkerk et al., 2002; Gilad et al., 2004) mediante un sistema de tres ecuaciones diferenciales que representaban respectivamente la dinámica espacio-temporal de la biomasa vegetal, del agua superficial y del agua en el suelo. Con estos modelos se investigó a fondo las implicaciones que para el ecosistema tiene la concentración de recursos bajo las plantas. En concreto, se vio que las comunidades vegetales son, gracias al feedback y a la acumulación del agua disponible bajo las plantas, capaces de mantener una mayor biomasa vegetal y de sobrevivir en condiciones más áridas que en ausencia de este mecanismo (Rietkerk et al., 2002). Por otro lado, se ha comprobado cómo las plantas pioneras en la formación de manchas de vegetación, "ingenieros del ecosistema" (Jones et al., 1994), son capaces de crear unas condiciones de mayor disponibilidad hídrica para que otras plantas puedan establecerse (Gilad et al., 2004). También se observó que las diferentes configuraciones espaciales presentan distintos grados de resiliencia y productividad (Yizhaq et al., 2005).

Posteriormente se han investigado otros mecanismos que no se tuvieron en cuenta inicialmente. En este sentido, destaca la inclusión de regímenes de precipitación realistas (Guttal y Jayaprakash, 2007), en contraposición con la precipitación uniforme de los 


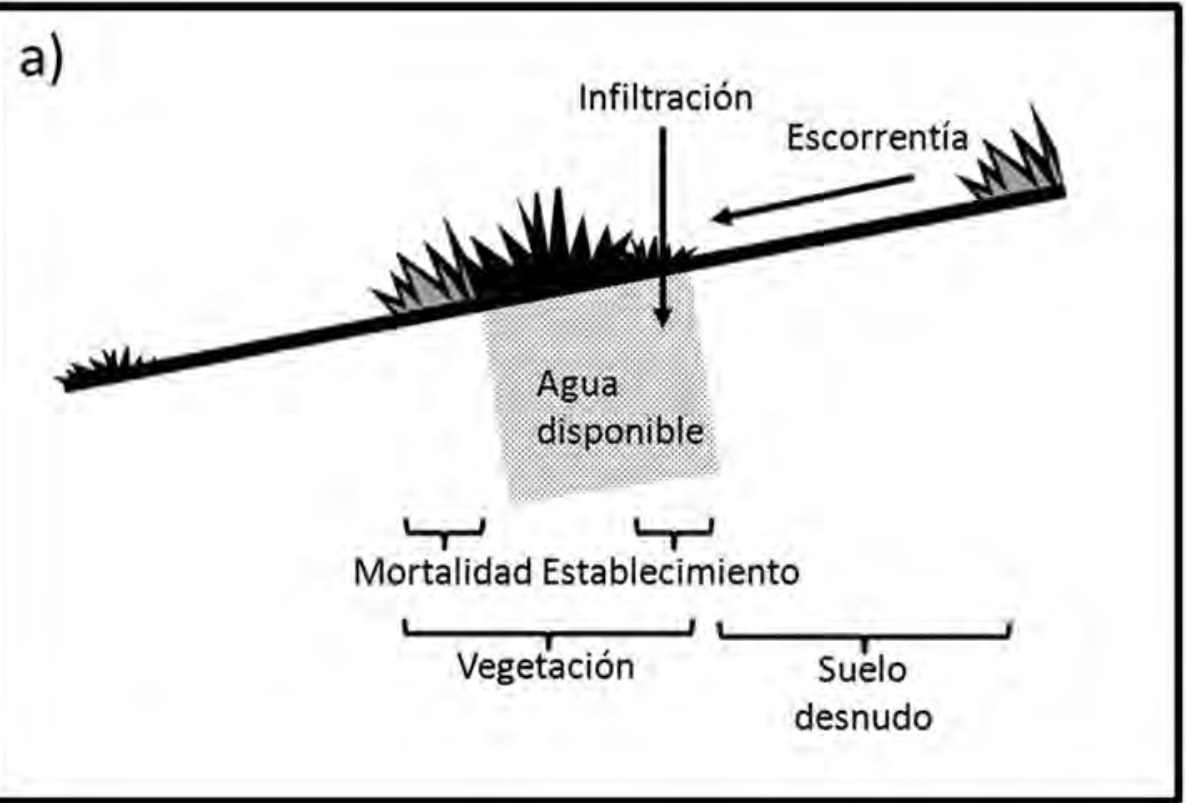

b)

\section{Infiltración}

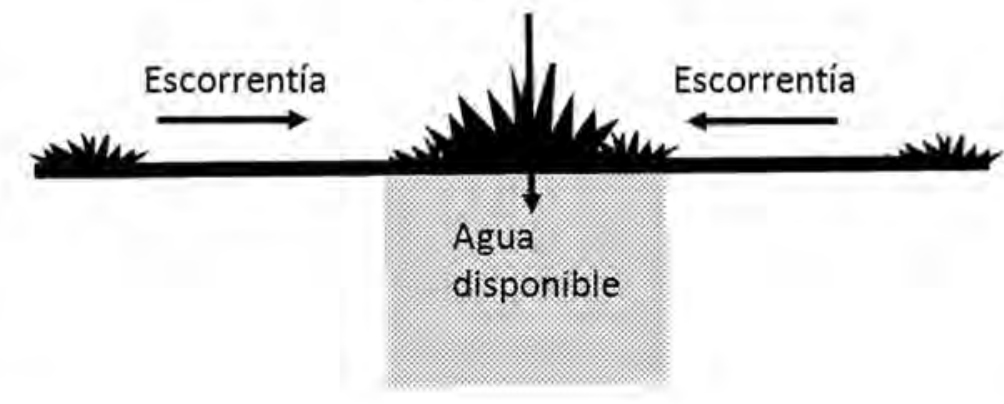

Figura 2. Esquema de los mecanismos ecohidrológicos responsables de la formación de patrones en la vegetación en a) laderas y $b$ ) condiciones sin pendiente.

modelos anteriores, con lo que se ha investigado cómo la variación estacional e interanual en el régimen de precipitaciones o los periodos prolongados de sequías juegan un papel importante en el estado final del ecosistema. También se ha descubierto que la estocasticidad en la variación interanual de las precipitaciones es uno de los factores responsables del mantenimiento de los patrones espaciales de la vegetación característicos de las zonas áridas y semi-áridas (D’Odorico et al., 2006b). 
Una simplificación frecuente de los modelos ecohidrológicos mencionados es la representación de la vegetación de una forma poco realista. En muchos modelos se modeliza la dispersión y establecimiento de nuevas plántulas con un proceso de difusión, en el que la biomasa vegetal se mueve de una celda a la contigua por un gradiente de biomasa. Aunque esta simplificación puede aceptarse para la vegetación con crecimiento clonal, como las gramíneas perennes (Murray, 2001), es poco realista para representar la dispersión por semillas, que puede alcanzar grandes distancias en especies anemócoras o zoócoras. Un avance en este sentido fueron los modelos que incorporaron la dispersión de semillas mediante una ecuación integro-diferencial, con la que la vegetación puede alcanzar una distancia considerable en cada dispersión, definida matemáticamente por una función de distribución de la biomasa que se dispersa (Pueyo et al., 2008; Thompson et al., 2008). Con estos modelos se obtienen patrones espaciales más heterogéneos que con los modelos que basan la dispersión en procesos de difusión, y permiten entender la implicación de las características reproductivas de las plantas (distancia de dispersión, habilidad en el establecimiento, etc.) en la formación de patrones espaciales y en la dinámica de los ecosistemas.

Otra simplificación frecuente de la representación de la vegetación en muchos modelos ecohidrológicos de zonas áridas es representarla como biomasa vegetal monoespecífica. Esto es un limitante para la aplicabilidad de los modelos ya que, en primer lugar, diferentes especies o grupos funcionales se pueden comportar de forma diferente frente a unas condiciones hídricas determinadas, y como consecuencia, el valor de los parámetros biológicos del modelo sería variable para cada tipo funcional. Además, las interacciones interespecíficas de facilitación y competencia son fundamentales para entender la dinámica y composición de las comunidades vegetales de los ecosistemas áridos y semi-áridos. Los modelos ecohidrológicos que tienen en cuenta explícitamente la interacción entre varios tipos de plantas abordan estas cuestiones. Gilad et al. (2007) desarrollaron un modelo de interacción entre vegetación leñosa y herbácea, con el que investigaron las interacciones entre las plantas (facilitación y competencia) en gradientes de estrés y de perturbaciones y a diferentes escalas espaciales, y comprobaron las hipótesis que planteaban las observaciones en ecosistemas reales, como la transición de competencia a facilitación y de nuevo a competencia a lo largo de un gradiente de aridez (Maestre et al., 2005). También se han investigado recientemente las interacciones bióticas entre gramíneas y matorrales a lo largo de gradientes de aridez (Pueyo et al., 2010), concluyendo que las diferentes características reproductivas de estas plantas (crecimiento clonal y dispersión por semillas a larga distancia respectivamente) proporcionan diferentes habilidades, siendo la dispersión de semillas clave a la hora de recuperarse desde un estado degradado, y confiriendo la reproducción clonal una gran resistencia frente a la aridez, debido al gran aprovechamiento que hacen las plantas con este tipo de reproducción de la concentración de recursos en torno a la vegetación preexistente (Pueyo et al., 2010). Por otro lado, la inclusión explícita de procesos fisiológicas de las plantas, como la fotosíntesis y la respiración en un modelo ecohidrológico permite evaluar el efecto neto de los cambios en temperatura, precipitación y $\mathrm{CO}_{2}$ predichos con el cambio climático (Kefi et al., 2008). 
Casi todos los modelos mencionados anteriormente son deterministas basados en ecuaciones diferenciales o integro-diferenciales. También los modelos ecohidrológicosestocásticos que simulan la dinámica vegetal de zonas áridas y semiáridas en cuadrículas (lattice) y cuya dinámica está basada en reglas de transición dependientes del entorno son abundantes (Thiery et al., 1995; Dunkerley, 1997a; Esteban y Fairen, 2006; Kéfi et al., 2007a). Una ventaja de estos modelos es que matemáticamente son más sencillos de implementar, y muy versátiles en los mecanismos que se incluyen (Vega y Montana, 2011). Aunque en algunos de ellos no se modeliza la dinámica del agua explícitamente, las reglas que gobiernan la dinámica de la vegetación están basadas, entre otras cosas, en la disponibilidad de agua para el establecimiento y crecimiento de las plantas. La flexibilidad que confieren estos modelos permite incorporar otros feedbacks simultáneamente, como las mejoras que acompañan a la presencia de plantas además de la mayor disponibilidad de agua (disminución de la radiación solar, mayor concentración de materia orgánica, etc.). Con un modelo estocástico basado en feedbacks entre la presencia de vegetación y las condiciones idóneas para que otras plantas se establezcan (Kéfi et al., 2007b), es decir, incluyendo la facilitación como una interacción biótica determinante, Kéfi et al. (2007a) comprobaron cómo cambia el patrón espacial de la vegetación Mediterránea en un gradiente de degradación cercano a la desertificación. También con modelos de autómatas celulares se ha comprobado el mayor papel que tienen las sequías respecto a la intensidad de pastoreo en el mantenimiento de la vegetación estructurada en bandas (Dunkerley, 1997b), y la productividad de la vegetación en diferentes configuraciones espaciales condicionadas por el régimen de precipitaciones (Esteban y Fairen, 2006). Recientemente se ha investigado con un modelo de autómatas celulares el efecto interactivo entre el régimen de precipitación, el pastoreo y las interacciones bióticas entre plantas en la formación de patrones vegetales (Vega y Montana, 2011), postulándose este modelo como una adecuada herramienta de gestión para los ecosistemas pastorales con este tipo de vegetación. Por su parte, con un complejo modelo combinando ecuaciones mecanicistas y dinámica en una cuadrícula, se investigó el efecto de la degradación de los patrones espaciales en la productividad del ecosistema (Ludwig et al., 1999).

Mención aparte merecen la abundancia de modelos ecohidrológicos, tanto determinísticos como estocásticos, centrados en explicar los patrones espaciales y la coexistencia de árboles y gramíneas en la sabana africana. En estos ecosistemas existe un feedback positivo entre la presencia de gramíneas y la infiltración de agua en el suelo. La coexistencia entre árboles y gramíneas se suele explicar por un mecanismo de partición del suelo en dos capas, una capa superficial, en la que gramíneas y árboles compiten por el agua, y una capa profunda a la que sólo acceden los árboles (Walker et al., 1981). Además, muchos modelos han demostrado que la inclusión del fuego y la herbivoría como procesos que abren huecos y permiten la colonización de plantas competitivamente inferiores es clave para explicar dicha coexistencia en situaciones climáticas o litológicas en las que sólo uno de los dos tipos de plantas dominaría (Dublín et al., 1990; Hochberg et al., 1994; van Langevelde et al., 2003; D'Odorico et al., 2006a; Baudena et al., 2010).

También existen numerosos modelos ecohidrológicos de zonas áridas y semiáridas más enfocados en predecir la productividad de la comunidad vegetal y la distribución 
del agua disponible, sin un componente espacial explicito, aunque con el feedback entre el agua y la biomasa vegetal incluido entre sus asunciones. Estos modelos permiten evaluar, entre otras cosas, los efectos del cambio climático en la disponibilidad de agua para las plantas (Tietjen et al., 2009), evaluar el efecto de diferentes tipos de plantas en la productividad de la comunidad vegetal y la distribución del agua disponible (Tietjen et al., 2009) y evaluar la sustentabilidad de ecosistemas pastorales frente al cambio climático (Lohmann et al., 2012).

\section{Conclusiones, aplicaciones y direcciones futuras}

La principal aportación de la modelización ecohidrológica al conocimiento del funcionamiento de los ecosistemas áridos y semi-áridos, y en concreto, de los modelos que incorporan feedbacks entre la disponibilidad de agua y la presencia de vegetación, es que permiten identificar los mecanismos responsables de la composición y estructura espacial que se observa en los ecosistemas reales. El estudio directo de los feedbacks ecohidrológicos requiere de manipulación experimental y de observaciones a largo plazo, además de estudios a varias escalas (Turnbull et al., 2012), por lo que los modelos se muestran muy útiles llegando a donde los experimentos y observaciones directas no alcanzan. Con modelos mecanicistas monoespécíficos se ha investigado el patrón espacial que produce la vegetación a lo largo de gradientes amplios de estrés (por ejemplo, de aridez y de pastoreo). Estos modelos son en gran medida simplificaciones de la realidad, pero su capacidad de predecir características fundamentales de ecosistemas presentes en todas las zonas áridas y semi-áridas (como el patrón espacial o la dinámica) aporta indicios de la generalidad y relevancia del feedback entre la presencia de vegetación y la disponibilidad de agua para las plantas. La incorporación de varios tipos de plantas, y otras características fisiológicas de la vegetación ha permitido refinar la relación entre la vegetación y la respuesta hidrológica de los ecosistemas, respondiendo a otras cuestiones más concretas, como la composición específica de la comunidad vegetal en determinadas condiciones abióticas o el papel que juegan las interacciones bióticas entre tipos de plantas en la estructura y dinámica de los ecosistemas. Por su parte, los modelos estocásticos han permitido incorporar otro tipo de feedbacks asociados al feedback entre la presencia de plantas y la disponibilidad de agua para las plantas, agrupados bajo el concepto "mecanismos de concentración de recursos bajo las plantas" (Rietkerk et al., 2004).

Una de las aplicaciones más relevantes de estos modelos es su capacidad para la predicción de los cambios bruscos en el estado de los ecosistemas, algo que va inherentemente asociados a los sistemas dominados por feedbacks (Rietkerk et al., 2004), por la tendencia de estos sistemas a permanecer en un estado hasta que cambian bruscamente a otro estado, aunque el cambio en el agente estresante haya sido gradual. Con la incorporación de los feedbacks a los modelos ecohidrológicos se ha podido investigar las implicaciones y los indicadores de estos cambios abruptos para los ecosistemas (Rietkerk et al., 2004; Zeng y Zeng, 2007), que no son sólo aplicables a las zonas áridas, sino que se trata de reglas de funcionamiento generales de los sistemas biológicos. 
Además, el conocimiento del funcionamiento de los ecosistemas dominados por feedbacks que ha aportado la modelización ha permitido la posterior aplicación del conocimiento generado a planes específicos de restauración de zonas áridas y semi-áridas permanentemente degradadas, que presentaban una degradación estable aun cuando los agentes estresantes externos (por ejemplo, el pastoreo o la eliminación brusca de la cubierta vegetal) habían sido eliminados (Suding et al., 2004). En un experimento realizado en el centro de la Depresión del Ebro, se aplicaron las teorías de los feedbacks entre presencia de vegetación y estado del suelo y del agua disponible para comparar varios métodos de restauración ecológica, llegando a la conclusión de que la cara y poco viable mejora directa de los suelos degradados y salinizados podría sustituirse por la menos costosa revegetación aprovechando las zonas de concentración de recursos (Pueyo et al. 2009).

Otra aplicación directa de estos modelos es la capacidad de incorporar la gestión pastoral al modelo, para predecir los recursos forrajeros disponibles para los animales y la dinámica a largo plazo bajo diferentes condiciones ambientales, algo especialmente relevante con la incertidumbre que sobre este tema pesa de cara al cambio climático (Lohmann et al., 2012). Muchas veces las fuerzas exógenas que provocan cambios de estado en los ecosistemas están fuera del alcance de actuación de los gestores (por ejemplo, el cambio climático). Sin embargo, los feedbacks que actúan como fuerzas estabilizadoras de los ecosistemas frente a las fuerzas exógenas sí que suelen estar al alcance de los gestores, por ejemplo, la conservación de la cubierta vegetal frente al sobrepastoreo (Turnbull et al., 2012).

Permanece como un reto para el futuro que los modelos ecohidrológicos que incorporan feedbacks sean capaces de discernir entre los diferentes mecanismos posibles que diferentes modelos proponen como relevantes para explicar la estructura y dinámica en las zonas áridas y semi-áridas. También es necesaria más investigación que conecte los estudios teóricos, con las observaciones de campo y a su vez ambos con las estrategias de gestión de los recursos de zonas áridas y semi-áridas (Rietkerk et al., 2004), de cara a preservar los ecosistemas a largo plazo frente a amenazas como el cambio climático o un uso inadecuado (por ejemplo, ganadero). En este sentido, parece que los modelos actuales no son totalmente adecuados para investigar los posibles cambios en los ecosistemas pastorales con el cambio climático (Tietjen y Jeltsch, 2007), debido a que no suelen incorporar en sus especificaciones suficientes detalles de los parámetros ambientales que conlleva el cambio climático, como niveles de $\mathrm{CO}_{2}$, o patrones de precipitación (aunque ver Kéfi et al., 2008; Guttal y Jayaprakash, 2007; Vega y Montaña, 2011). Dada la relevancia ecológica y socioeconómica de predecir la dinámica de los ecosistemas pastorales con el cambio climático, esto permanece como un reto para el futuro en la investigación con modelos ecohidrológicos.

\section{Agradecimientos}

Este artículo tiene el soporte económico de los proyectos GA-LC-020/2010 y 2012/GA-LC074. Agradezco a Rubén Díaz-Sierra sus comentarios, que han contribuido a mejorar este artículo. 


\section{Referencias bibliográficas}

Arnau-Rosalen, E., Calvo-Cases, A., Boix-Fayos, C., Lavee, H., Sarah, P. 2008. Analysis of soil surface component patterns affecting runoff generation. An example of methods applied to Mediterranean hillslopes in Alicante (Spain). Geomorphology 101, 595-606.

Barbier, N., Couteron, P., Lefever, R., Deblauwe, V., Lejeune, O. 2008. Spatial decoupling of facilitation and competition at the origin of gapped vegetation patterns. Ecology 89, 1521-1531.

Baudena, M., D’Andrea, F., Provenzale, A. 2010. An idealized model for tree-grass coexistence in savannas: the role of life stage structure and fire disturbances. Journal of Ecology 98, 74-80.

Bautista, S., Mayor, A.G., Bourakhouadar, J., Bellot, J. 2007. Plant spatial pattern predicts hillslope semiarid runoff and erosion in a Mediterranean landscape. Ecosystems 10, 987-998.

Bedford, D.R., Small, E.E. 2008. Spatial patterns of ecohydrologic properties on a hillslope-alluvial fan transect, central New Mexico. Catena 73, 34-48.

Bergkamp, G., Cerdà, A., Imeson, A.C. 1999. Magnitude-frequency analysis of water redistribution along a climate gradient in Spain. Catena 37, 129-146.

Bhark, E.W., Small, E.E. 2003. Association between plant canopies and the spatial patterns of infiltration in shrubland and grassland of the Chihuahuan Desert, New Mexico. Ecosystems 6, 185-196.

Bochet, E., Rubio, J.L., Poesen, J. 1999. Modified topsoil islands within patchy Mediterranean vegetation in SE Spain. Catena 38, 23-44.

Borgogno, F., D’Odorico, P., Laio, F., Ridolfi, L. 2009. Mathematical models of vegetation pattern formation in ecohydrology. Reviews of Geophysics 47, RG1005.

Callaway, R.M. 2007. Positive interactions and interdependence in plant communities. Springer, Dordrecht, $415 \mathrm{pp}$.

Castellano, M.J., Valone, T.J. 2007. Livestock, soil compaction and water infiltration rate: Evaluating a potential desertification recovery mechanism. Journal of Arid Environments 71, 97-108.

Cerdà, A. 1997. The effect of patchy distribution of Stipa tenacissima L. on runoff and erosion. Journal of Arid Environments 36, 37-51.

Couteron, P., Lejeune, O. 2001. Periodic spotted patterns in semi-arid vegetation explained by a propagation-inhibition model. Journal of Ecology 89, 616-628.

D'Odorico, P., Laio, F., Ridolfi, L. 2006a. A probabilistic analysis of fire-induced tree-grass coexistence in savannas. American Naturalist 167, E79-E87.

D’Odorico, P., Laio, F., Ridolfi, L. 2006b. Vegetation patterns induced by random climate fluctuations. Geophysical Research Letters 33, L19404.

Dublin, H.T., Sinclair, A.R.E., McGlade, J. 1990. Elephants and fire as causes of multiple states in the Serengeti-Mara woodlands. Journal of Animal Ecology 59, 1147-1164.

Dunkerley, D.L. 1997a. Banded vegetation: Development under uniform rainfall from a simple cellular automaton model. Plant Ecology 129, 103-111.

Dunkerley, D.L. 1997b. Banded vegetation: Survival under drought and grazing pressure based on a simple cellular automaton model. Journal of Arid Environments 35, 419-428.

Dunne, T., Western, D., Dietrich, W.E. 2011. Effects of cattle trampling on vegetation, infiltration, and erosion in a tropical rangeland. Journal of Arid Environments 75, 58-69.

Eldridge, D.J., Greene, R.S.B. 1994. Microbiotic soil crusts - A review of their roles in soil and ecological processes in the rangelands of Australia. Australian Journal of Soil Research 32, 389-415.

Eldridge, D.J., Zaady, E., Shachak, M. 2000. Infiltration through three contrasting biological soil crusts in patterned landscapes in the Negev, Israel. Catena 40, 323-336. 
Eldridge, D.J., Bowker, M.A., Maestre, F.T., Alonso, P., Mau, R.L., Papadopoulos, J., Escudero, A. 2010. Interactive effects of three ecosystem engineers on infiltration in a semi-arid Mediterranean grassland. Ecosystems 13, 499-510.

Esteban, J., Fairen, V. 2006. Self-organized formation of banded vegetation patterns in semi-arid regions: A model. Ecological Complexity 3, 109-118.

Gilad, E., Von Hardenberg, J., Provenzale, A., Shachak, M., Meron, E. 2004. Ecosystem engineers: from pattern formation to habitat creation. Physical Review Letters 93, 098105.

Gilad, E., von Hardenberg, J., Provenzale, A., Shachak, M., Meron, E. 2007. A mathematical model of plants as ecosystem engineers. Journal of Theoretical Biology 244, 680-691.

Greigsmith, P. 1979. Pattern in vegetation. Journal of Ecology 67, 755-779.

Guttal, V., Jayaprakash, C. 2007. Self-organization and productivity in semi-arid ecosystems: Implications of seasonality in rainfall. Journal of Theoretical Biology 248, 490-500.

Hamerlynck, E.P., McAuliffe, J.R., McDonald, E.V., Smith, S.D. 2002. Ecological responses of two Mojave Desert shrubs to soil horizon development and soil water dynamics. Ecology 83, 768-779.

HilleRisLambers, R., Rietkerk, M., van den Bosch, F., Prins, H.H.T., de Kroon, H. 2001. Vegetation pattern formation in semi-arid grazing systems. Ecology 82, 50-61.

Hochberg, M.E., Menaut, J.C., Gignoux, J. 1994. The influences of tree biology and fire in the spatial structure of the West African savannah. Journal of Ecology 82, 217-226.

Huxman, T.E., Wilcox, B.P., Breshears, D.D., Scott, R.L., Snyder, K.A., Small, E.E., Hultine, K., Pockman, W.T., Jackson, R.B. 2005. Ecohydrological implications of woody plant encroachment. Ecology 86, 308-319.

Joffre, R., Rambal, S. 1993. How tree cover influences the water balance of Mediterranean rangelands. Ecology 74, 570-582.

Jones, C.G., Lawton, J.H., Shachak, M. 1994. Organisms as ecosystem engineers. Oikos 69, 373-386.

Kéfi, S., Rietkerk, M., Alados, C.L., Pueyo, Y., Papanastasis, V.P., ElAich, A., De Ruiter, P.C. 2007a. Spatial vegetation patterns and imminent desertification in Mediterranean arid ecosystems. Nature 449, 213-217.

Kéfi, S., Rietkerk, M., van Baalen, M., Loreau, M. 2007b. Local facilitation, bistability and transitions in arid ecosystems. Theoretical Population Biology 71, 367-379.

Kefi, S., Rietkerk, M., Katul, G.G. 2008. Vegetation pattern shift as a result of rising atmospheric $\mathrm{CO}(2)$ in arid ecosystems. Theoretical Population Biology 74, 332-344.

Kéfi, S., Alados, C.L., Chaves, R.C.G., Pueyo, Y., Rietkerk, M. 2010. Is the patch size distribution of vegetation a suitable indicator of desertification processes? Comment. Ecology 91, 3739-3742.

Klausmeier, C.A. 1999. Regular and irregular patterns in semiarid vegetation. Science 284, 1826-1828.

Lefever, R., Lejeune, O. 1997. On the origin of tiger bush. Bulletin of Mathematical Biology 59, 263-294.

Lejeune, O., Tlidi, M. 1999. A model for the explanation of vegetation stripes (tiger bush). Journal of Vegetation Science 10, 201-208.

Lejeune, O., Couteron, P., Lefever, R. 1999. Short range co-operativity competing with long range inhibition explains vegetation patterns. Acta Oecologica-International Journal of Ecology 20, 171-183.

Lohmann, D., Tietjen, B., Blaum, N., Joubert, D.F., Jeltsch, F. 2012. Shifting thresholds and changing degradation patterns: climate change effects on the simulated long-term response of a semi-arid savanna to grazing. Journal of Applied Ecology 49, 814-823. 
Ludwig, J.A., Tongway, D.J., Marsden, S.G. 1999. Stripes, strands and stipples: modelling the influence of three landscape banding patterns on resource capture and productivity in semiarid woodlands, Australia. Catena 37, 257-273.

Ludwig, J.A., Wilcox, B.P., Breshears, D.D., Tongway, D.J., Imeson, A.C. 2005. Vegetation patches and runoff-erosion as interacting ecohydrological processes in semiarid landscapes. Ecology 86, 288-297.

Maestre, F.T., Escudero, A. 2009. Is the patch size distribution of vegetation a suitable indicator of desertification processes? Ecology 90, 1729-1735.

Maestre, F.T., Huesca, M., Zaady, E., Bautista, S., Cortina, J. 2002. Infiltration, penetration resistance and microphytic crust composition in contrasted microsites within a Mediterranean semi-arid steppe. Soil Biology \& Biochemistry 34, 895-898.

Maestre, F.T., Cortina, J., Bautista, S., Bellot, J., Vallejo, R. 2003. Small-scale environmental heterogeneity and spatiotemporal dynamics of seedling establishment in a semiarid degraded ecosystem. Ecosystems 6, 630-643.

Maestre, F.T., Valladares, F., Reynolds, J.F. 2005. Is the change of plant-plant interactions with abiotic stress predictable? A meta-analysis of field results in arid environments. Journal of Ecology 93, 748-757.

MARM 2008. Programa de acción nacional contra la desertificación. Ministerio de Medio Ambiente, Medio Rural y Marino. Disponible en: http://www.marm.es.

Millennium Ecosystem Assessment 2005. Ecosystems and human well-being: desertification synthesis. World Resources Institute, Washingon, DC.

Mills, A., Fey, M., Donaldson, J., Todd, S., Theron, L. 2009. Soil infiltrability as a driver of plant cover and species richness in the semi-arid Karoo, South Africa. Plant and Soil 320, 321-332.

Moret, D., Pueyo, Y., Bueno, C.G., Alados, C.L. 2011. Hydro-physical responses of gypseous and non-gypseous soils to livestock grazing in a semi-arid region of NE Spain. Agricultural Water Management 98, 1822-1827.

Murray, J.D. 2001. Mathematical Biology, II: Spatial Models and Biomedical Applications. Springer, New York.

Noy-Meir, I. 1973. Desert ecosystems: environment and producers. Annual Review of Ecology and Systematics 4, 25-51.

Paruelo, J.M., Sala, O.E. 1995. Water losses int he Patagonian steppe: a modelling approach. Ecology 76, 510-520.

Pueyo, Y., Kéfi, S., Alados, C.L., Rietkerk, M. 2008. Dispersal strategies and spatial organization of vegetation in arid ecosystems. Oikos 117, 1522-1532.

Pueyo, Y., Alados, C.L., García-Ávila, B., Kéfi, S., Maestro, M., Rietkerk, M. 2009. Comparing direct abiotic amelioration and facilitation as tools for restoration of semi-arid grassland. Restoration Ecology 17, 908-916.

Pueyo, Y., Kéfi, S., Díaz-Sierra, R., Alados, C.L., Rietkerk, M. 2010. The role of reproductive plant traits and biotic interactions in the dynamics of semi-arid plant communities. Theoretical Population Biology 78, 289-297.

Puigdefabregas, J., Solé, A., Gutiérrez, L., del Barrio, G., Boer, M. 1999. Scales and processes of water and sediment redistribution in drylands: results from the Rambla Honda field site in Southeast Spain. Earth-Science Reviews 48, 39-70.

Reynolds, J.F., Stafford Smith, D.M., Lambin, E.F., Turner, B.L., Mortimore, M., Batterbury, S.P.J., Downing, T.E., Dowlatabadi, H., Fernandez, R.J., Herrick, J.E., Huber-Sannwald, E., Jiang, H., Leemans, R., Lynam, T., Maestre, F.T., Ayarza, M., Walker, B. 2007. Global desertification: Building a science for dryland development. Science 316, 847-851.

Rietkerk, M., Van de Koppel, J. 2008. Regular pattern formation in real ecosystems. Trends in Ecology \& Evolution 23, 169-175. 
Rietkerk, M., Boerlijst, M.C., van Langevelde, F., HilleRisLambers, R., van de Koppel, J., Kumar, L., Prins, H.H.T., de Roos, A.M. 2002. Self-organization of vegetation in arid ecosystems. American Naturalist 160, 524-530.

Rietkerk, M., Dekker, S.C., de Ruiter, P.C., van de Koppel, J. 2004. Self-organized patchiness and catastrophic shifts in ecosystems. Science 305, 1926-1929.

Rodríguez-Iturbe, I., Porporato, A. 2004. Ecohydrology of water-controlled ecosystems: soil moisture and plant dynamics. Cambridge University Press, New York.

Rodríguez-Iturbe, I., Porporato, A., Laio, F., Ridolfi, L. 2001. Plants in water-controlled ecosystems: active role in hydrological processes and responses to water stress, I. Scope and general outline. Advances in Water Resources 24, 697-705.

Segoli, M., Ungar, E.D., Shachak, M. 2008. Shrubs enhance resilience of a semi-arid ecosystem by engineering and regrowth. Ecohydrology 1, 330-339.

Shachak, M., Sachs, M., Moshe, I. 1998. Ecosystem management of desertified shrublands in Israel. Ecosystems 1, 475-483.

Suding, K.N., Gross, K.L., Houseman, G.R. 2004. Alternative states and positive feedbacks in restoration ecology. Trends in Ecology and Evolution 19, 46-53.

Thiery, J.M., Dherbes, J.M., Valentin, C. 1995. A model simulating the genesis of banded vegetation patterns in Niger. Journal of Ecology 83, 497-507.

Thompson, S., Katul, G., McMahon, S.M. 2008. Role of biomass spread in vegetation pattern formation within arid ecosystems. Water Resources Research 44, W10421.

Tietjen, B., Jeltsch, F. 2007. Semi-arid grazing systems and climate change: a survey of present modelling potential and future needs. Journal of Applied Ecology 44, 425-434.

Tietjen, B., Zehe, E., Jeltsch, F. 2009. Simulating plant water availability in dry lands under climate change: A generic model of two soil layers. Water Resources Research 45, W01418.

Tongway, D.J., Valentin, C., Seghieri, J. 2001. Banded vegetation patterning in arid and semiarid environments. Springer-Verlag, New York.

Turing, A.M. 1990. The chemical basis of morphogenesis. 1953. Bulletin of Mathematical Biology 52, 153-197.

Turnbull, L., Wilcox, B.P., Belnap, J., Ravi, S., D’Odorico, P., Childers, D., Gwenzi, W., Okin, G., Wainwright, J., Caylor, K.K., Sankey, T. 2012. Understanding the role of ecohydrological feedbacks in ecosystem state change in drylands. Ecohydrology 5, 174-183.

Valentin, C., d'Herbès, J.M., Poesen, J. 1999. Soil water components of banded vegetation patterns. Catena 37, 1-24.

Van Langevelde, F., van de Vijver, C., Kumar, L., van de Koppel, J., de Ridder, N., van Andel, J., Skidmore, A.K., Hearne, J.W., Stroosnijder, L., Bond, W.J., Prins, H.H.T., Rietkerk, M. 2003. Effects of fire and herbivory on the stability of savanna ecosystems. Ecology 84, 337-350.

Vega, E., Montana, C. 2011. Effects of overgrazing and rainfall variability on the dynamics of semiarid banded vegetation patterns: A simulation study with cellular automata. Journal of Arid Environments 75, 70-77.

Von Hardenberg, J., Meron, E., Shachak, M., Zarmi, Y. 2001. Diversity of vegetation patterns and desertification. Physical Review Letters 87, 198101.

Walker, B.H., Ludwig, D., Holling, C.S., Peterman, R.M. 1981. Stability of semi-arid savannah grazing systems. Journal of Ecology 69, 473-498.

West, N.E. 1990. Structure and function of microphytic soil crust in wildland ecosystems of arid to semi/arid regions. Advances in Ecological Research 20, 180-223.

Yizhaq, H., Gilad, E., Meron, E. 2005. Banded vegetation: biological productivity and resilience. Physica A: Statistical Mechanics and its Applications 356, 139-144.

Zeng, X., Zeng, X. 2007. Transition and pattern diversity in arid and semiarid grassland: A modeling study. Journal of Geophysical Research-Biogeosciences 112, G04008. 